\title{
Digital Watermark of 3D CAD Product Model
}

\author{
Xiaoqing Feng, Yanan Liu and Lincong Fang \\ College of Information, Zhejiang University of Finance \& Economics, Hangzhou, \\ 310018, China \\ fxq_snake@163.com
}

\begin{abstract}
This paper proposes a novel watermarking algorithm of $3 D$ CAD product model, which is able to keep strictly geometrical shape around embedding watermark and make watermarked model have function transparency. Firstly, the isolated geometrical entities are selected as watermark carriers. Secondly, the watermark is embedded into the isolated geometrical entities with a geometric entity substitution algorithm. When extracting the watermark, the original product model and pre-process (registration or re-sampling) need not be provided. The experimental results have shown that the proposed watermarking algorithm is robustness against some common attacks, such as transformation, rotation, scaling and entity deletion.
\end{abstract}

Keywords: 3D CAD product model, watermark, isolated geometric entity

\section{Introduction}

Watermarking can be classified into two categories [S. Kanai, H. Date, and T. Kishinami, 1998]: blind watermark and non-blind one. For blind watermark, the original model is not required during extraction. Non-blind watermark, which is also called the private watermark, needs original model during extraction. Many watermarking algorithms have been proposed and copyright technologies of image and video have also been widely elaborated. However, 3D watermarking technique is relatively new, and is focused mainly on 3D mesh model, such as frequency domain algorithms [H.K. Chen and Y.H. Chen, 2010][ L. Li, D. Zhang, Z.G. Pan, J.Y. Shi, K. Zhou, and K. Ye, 2004] and spatial domain method [A.G. Bors,2006][ Y.P. Wang and S.M. Hu,2009][W. Kai, L. Guillaume, D. Florence, and B. Atilla,2011]. Nevertheless, 3D CAD product model instead of 3D mesh model is widely used in the practical industrial design and manufacturing fields. Compared with 3D mesh model, there are some differences as follows: Firstly, 3D mesh model algorithms are based on polygon meshes, while 3D CAD product model is sliced up into layers, which means that it is made up of one or more parts and a part may consists of many features. So, many 3D mesh watermarking algorithms cannot be directly applied in 3D CAD product model. Secondly, the difference between mesh model and CAD product model is that the geometric detail of mesh model need not be expressed exactly. Thus, the watermark can be embedded by adjusting geometric topology information of mesh model. On the contrary, CAD product model must keep the exact geometrical shape of model because of the tolerance requirements of machining technology. Watermarking of 3D product CAD model keeps not only imperceptibility of geometrical shape but also function transparency. Thirdly, 3D mesh model is a collection of vector and polygonal facets, which contain a great deal of redundant information, while $3 \mathrm{D}$ product $\mathrm{CAD}$ model is a vector model, whose geometrical feature is curve surface and NURBS, which doesn't provide enough redundant information and embed it into the watermark. Therefore, existing 3D mesh watermarking algorithms cannot be transplanted directly into 3D CAD product model. It is necessary to explore the copyright technologies of 3D CAD product model. To the best 
of our knowledge, little research pays attention to watermarking of 3D CAD product model compared to 3D mesh model and 2D CAD engineering drawing. This paper presents a high-fidelity digital watermarking algorithm for 3D CAD product model according to the requirements of preserving the exact geometrical shape. The watermark is embedded into the isolated geometrical entities using geometric entity substitution. When extracting the watermark, the original product model and pre-process (registration or re-sampling) need not be provided. And watermark is robustness against some common attacks. Our work is an initial exploration to protect the copyright of 3D CAD product model. It provides an effective watermarking scheme by embedding directly the watermark into the 3D product model for collaborative manufacturing systems.

The remainder of this paper is organized as follows. "Section 2" surveys the related works. "Section 3" describes the data characteristics of 3D CAD product model and some common attack types. Watermarking algorithm of 3D CAD product model is described in detail in Section 4. Section 5 presents some experimental results. Finally, in Section 6, we give the summary and conclusions.

\section{Related Works}

\subsection{Watermarking for 3D Mesh Models}

Ohbuchi et al. firstly proposed 3D mesh model watermark algorithm [R. Ohbuchi, H. Masuda, and M. Aono, 1997]. However, these early watermark algorithms have not strong robustness. Recently, 3D digital watermark techniques are developed quickly. Ai et al. [Q.S. Ai, Q. Liu, Z.D. Zhou, L. Yang, and S.Q, 2009] introduced a 3D mesh model watermark based on Voronoi patches segmentation. Then, DCT (Discrete Cosine Transform) [R.H. Juan, A. Martion, and P.G. Fernando, 2000] is employed to embed watermarking into range images. This watermarking based on frequency technique is able to resist against complicated attacks, such as mesh connectivity attacks (cropping, re-meshing, subdivision and simplification) and signal attacks (noising, smoothing and vertex coordinate quantization), etc. However, these watermarking algorithms require the original model and complicated pre-processing (registration or re-sampling), which affect the quality of the extracted watermark and make the non-blind watermarking impractical. In [ J.W. Cho, R. Prost, and H.Y. Jung,2007], Cho et al. gave a blind mesh watermarking scheme, which has been considered as the better robust blind spatial scheme proposed so far. The histogram of the distances between vertices and mesh gravity center is firstly constructed, and then the distribution of the mesh vertex norms is modified to hide a multi-bit watermark. However, it is very vulnerable to cropping attacks. We proposed a double-watermark mesh algorithm based on feature segmentation and redundancy information [X.Q. Feng, W.Y. Zhang, and Y.N. Liu, 2012]. This method can effectively resist against cropping.

\subsection{Watermarking for CAD Product Data}

Mesh models are always considered as a kind of occasional model during industrial design. Many engineers and enterprises use some CAD softwares to design product model, such as 2D CAD engineering graphics or 3D CAD entity models. These CAD products data can also be easily duplicated and redistributed without any loss of quality along the Web. Therefore, copyright is an important issue in a CAD product.

CAD products include 2D CAD engineering graphics and 3D CAD product models. In 2D CAD engineering watermarking, Kwon firstly proposed digital watermarking for CAD drawings using a self-adaptive algorithm related to the length of lines, angles of arcs, and radius of circles [K.R. Kwon, H.J. Chang, G.S. Jung, K.S. Moon, and S.H. Lee, 2006]. Cai et al. presented a watermarking algorithm based on the orthogonal complete U-system [Z.C. Cai, W. Sun, C.Z. Xiong, and D.X. Qi, 2005]. Although these methods 
are robust against geometrical transformation, it cannot resist attacks of entity addition and deletion. Huang et al. proposed a capacity variable watermarking algorithm based on Complex Number System [X.F. Huang, F. Peng, and T. Deng, 2008]. The coordinates of vertices in $2 \mathrm{D}$ engineering graphic are embedded into watermark, which is transformed into Gaussian integer. It is robust against geometric transformation, entity addition and deletion, but it is non-blind and original graphic should be provided when watermarking is extracted. Peng presented a reversible watermarking scheme for two dimensional CAD engineering graphics based on improved difference expansion [F. Peng, Y.Z. Lei, M. Long, and X.M. Sun, 2011]. It has a large capacity, good imperceptibility, and is robust against operations such as transformation, rotation, and equal scaling.

Although most of watermarking algorithms focus on 2D CAD engineering graphics, there are a few researches on 3D CAD product model. Kwon proposed a 3D CAD drawing watermarking based on three components that are line, arc, and 3Dface. The watermark is embedded into the length in Line, the radius of curvature in Arc, and the length ratio of two sides in 3DFACE. It can resist against format conversion, layer cutting and geometrical attacks. However, the scheme requires the index and order of embedding components. So, watermark will be extracted unsuccessfully if it suffers from component rearrangement. Lee introduced an improved 3D CAD drawing watermarking scheme [S.H. Lee and K.R. Kwon, 2010] using k-means++ clustering based on Kwon's algorithm. This scheme is more robust against file format conversion, layer attacks, component rearrangement, and geometrical attacks. Although Kwon and Lee proposed 3D CAD watermarking schemes, these watermarking algorithms are fit only for CAD drawings based on vector data and cannot deal with product models based on feature modeling. Moreover, designing product models based on feature modeling is the main method in the industry. And we make an initial exploration to protect product model based on feature modeling.

The proposed watermarking scheme in this paper presents some similarities with the work of Kwon et al. and Lee, et al.. We all select Lines and Circular Arc entities to embed watermarks. The key difference between our method and the above two algorithms lies in geometric entity selecting techniques, and the use of a novel embedding scheme based on geometrical entity substituted. Kwon randomly selected geometrical entities, Lee applied k-means++ clustering to select entities. In order to keep geometrical topological structure, we select isolated geometrical entity to embed watermark. Furthermore, it is important that CAD product model must keep strictly geometrical shape around embedding watermark. To attain this goal, we use an entity substitution scheme instead of typical method of adjusting coordinate of vertexes.

\section{Data Characteristics of 3D CAD Product Model and Attack Types}

Watermarking can also be classified into two categories: fragile watermarking and robust watermarking. Fragile watermarking is very susceptible to change of host signal and it is mainly used for the detection of manipulation. Robust watermarking is robust against attacks, either accidentally or intentionally. It is mainly used in copyright protection.

Watermarks are embedded into geometrical features of 3D CAD product model for the purpose of copyright protection. In industrial world, 3D CAD product model is constructed using three-dimensional modeling software, such as ProE, Solidworks and UG, which can express the design idea more intuitively than 2D engineering drawing. During product design process, CAD model is made up of a lot of geometrical entities, such as Lines, Circular Arc, B-Spline Curve, etc. Furthermore, a product model can be represented in various data formats such as IGES, STEP, 3DS and STL. IGES is the most general file format and a CAD user can exchange product data models among Computer-aided design (CAD) systems using IGES file. This paper uses IGES format as 
3D CAD prodcut model file. And we select Lines and Circular Arcs to embed watermarks. This section describes the structure of the IGES file structure and common attack types.

\subsection{Common Attack Types}

The attacks constitute a critical factor when designing 3D CAD product model watermarking algorithms. Lee and Kwon have discussed some common types of attack in. When designing a CAD model, engineers often use some operations, such as translation, rotation, scaling, copying, deleting, etc. Among these operations, translation, rotation, scaling and combinations of these three operations, are called similarity transformations and also are the most common attacks.

Similarity Transformation: Similarity transformations include translation, rotation, scaling and combinations of these three operations. They are the most common geometric attacks, and engineers often use some of these operations to design a CAD model

Geometrical Entity Attack: Generally speaking, geometric entities can be added or deleted deliberately. This kind of attack is quite difficult to handle. Specifically, entity deletion will change the topology of the 3D CAD product model.

File Format Conversion: Sometimes, a 3D CAD product model can be stored or converted to some formats, such as IGES or STEP. So, 3D CAD product data will encounter the problem of file format conversion.

\section{3D CAD Product Model Watermarking}

Our new algorithm is described in this paper with the following pipelines.

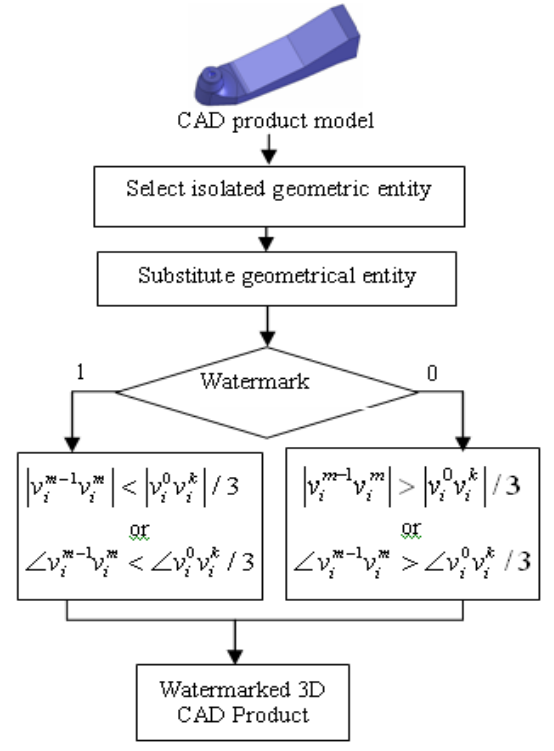

(a)

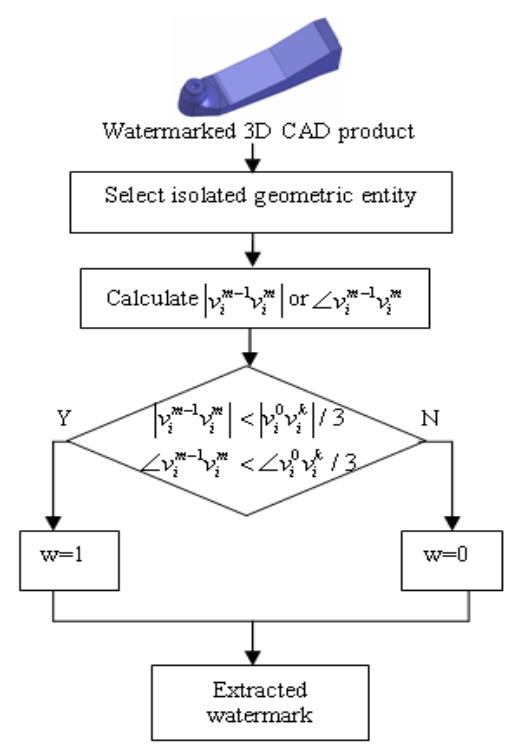

(b)

Figure 1. Pipeline of Watermark Embedding and Extraction $(2 \leq m \leq k-1, \mathbf{k}=\mathbf{3})$

Figure 1 (a) illustrates the block diagram of our blind watermark embedding procedure. Firstly, some geometrical entities, which are Line or Arc entities, are selected for embedding watermark. In this scheme, in order to prevent the geometrical shape of CAD products, which need embed watermarks, from any distortion to CAD product models, a substitution algorithm of geometrical entity is employed. Line or Arc entity is substituted with 3 Line or Arc entities. At last, embed watermark into CAD product models through adjusting length of line or angle of arc. Figure 1 (b) illustrates the block diagram of our 
blind watermark extraction procedure. Watermarks are extracted according to the proportion of line or arc.

\subsection{Isolated Geometric Entity and Geometric Entity Substitution}

A CAD product model is made up of many entities. In order to express and describe the exact model, entities always quote each other. There are some fundamental entities that can be used to embed watermarks, such as Line entity, Arc entity and Curve Entity. In our watermarking algorithm, Line entity and Arc entity are processed to embed watermark. And the watermark is embedded into the model by substituting an original geometric entity with three same types of geometric entities. A line entity is substituted with 3 three Line entities and an Arc entity is substituted with 3 three Arc entities. However, Type 120 entity is a kind of revolution surface and is defined with an axis of rotation and generatrix entity. If the axis of rotation or generatrix entity is modified to embed watermark, it will bring about incomplete model. So, we need to select isolated geometric entities to substitute and embed watermark.

Isolated geometric entity: It is Line entity or Arc entity, which is not quoted by a surface of revolution (Type 120 entity).

In a CAD product model, the geometrical shape of the model must be kept consistent. Typical watermarking methods, e.g., adjusting slightly the coordination of vertexes are not fitted to CAD product models. So, a method of geometrical entity substitution is applied in our watermarking scheme. A line or arc entity is substituted with many line or arc entities. Take line entity substituted for instance. An original line entity $v_{0} v_{l}$ is substituted with three Line entities $v_{0} v_{1}, v_{1} v_{2}$ and $v_{2} v_{3}$, as shown in Fig. 2. This approach of entity substitution would not affect the data accuracy and quality of CAD product model. Furthermore, points $v_{1}$ and $v_{2}$ moving along Line $v_{0} v_{3}$ would not bring any influence on data analysis of CAD product model as well.

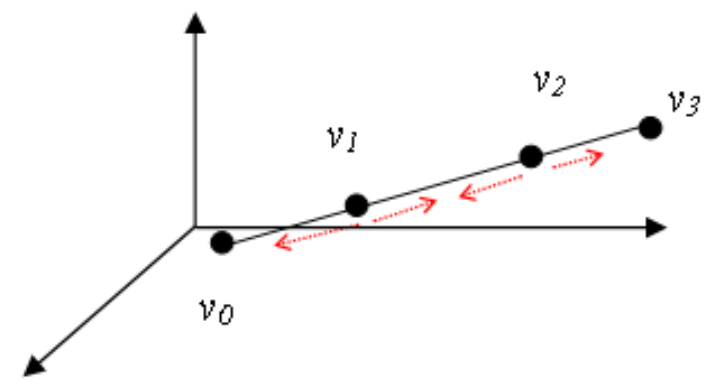

Figure 1. Moving along the Original Geometrical Entity

The method, which segments the Line or Arc, and is used to fit a given geometrical entity $v_{i}^{0} v_{i}^{k}$, is described as following:

Step 1: the i geometrical entity is noted as $v_{i}^{0} v_{i}^{k}$, then $v_{i}^{0} v_{i}^{k}$ is divided into equally three parts using additional points $v_{i}^{m}, 2 \leq m \leq k-1, \mathrm{k}=3$.

Step 2: delete original geometrical entity $v_{i}^{0} v_{i}^{k}$

Step 3: draw geometrical entities $v_{i}^{0} v_{i}^{1}, \ldots, v_{i}^{m} v_{i}^{k}, 2 \leq m \leq k-1, \mathrm{k}=3$.

\subsection{Watermark Embedding}

The watermark embedding scheme is implemented by moving the location of $v_{i}^{m}$ along the geometrical entity $v_{i}^{0} v_{i}^{k}$ according to each watermark bit. The embedding methods 
for line and arc entities are similar, but the embedding conditions to be satisfied are different. Length of new line entity $v_{i}^{0} v_{i}^{k}$ is considered when embedding watermark into Line entity. For Arc entity, we mainly take into account the angle of $\operatorname{Arc} v_{i}^{0} v_{i}^{k}$

4.2.1 Embedding Watermark Bits into Line Entity: In order to keep the shape of CAD product model, vertexes' coordinates $v_{i}^{1}$ and $v_{i}^{2}$ of line entity $v_{i}^{0} v_{i}^{3}$ are not allowed to be modified. So, we need to add redundant information for meeting the requirements of embedding watermark. The algorithm of geometrical entity substitution introduced above can solved this problem perfectly. Line entity $v_{i}^{0} v_{i}^{3}$ is evenly divided into three line entities by carrying out the algorithm of geometrical entity substitution, as shown in Figure 3.

Step 1: calculate coordinates of additional redundant points $v_{i}^{1}\left(v_{1 x}, v_{1 y}, v_{1 z}\right)$ and $v_{i}^{2}\left(v_{2 x}, v_{2 y}, v_{2 z}\right)$.

$$
v_{1 x}=\frac{2 v_{0 x}+v_{3 x}}{3} \quad, \quad v_{1 y}=\frac{2 v_{0 y}+v_{3 y}}{3} \quad, \quad v_{1 z}=\frac{2 v_{0 z}+v_{3 z}}{3}
$$

$$
v_{2 x}=\frac{v_{0 x}+2 v_{3 x}}{3} \quad, \quad v_{2 y}=\frac{v_{0 y}+2 v_{3 y}}{3} \quad, \quad v_{2 z}=\frac{v_{0 z}+2 v_{3 z}}{3}
$$

Step2: adjust the location of $v_{i}^{2^{\prime}}\left(v_{2 x}^{\prime}, v_{2 y}^{\prime}, v_{2 z}^{\prime}\right)$ according to watermarking bit w(i) using the following equations.

$$
v_{2 x}^{\prime}=\frac{v_{0 x}+v_{3 x}}{2}, \quad v_{2 y}^{\prime}=\frac{v_{0 y}+v_{3 y}}{2}, \quad v_{2 z}^{\prime}=\frac{v_{0 z}+v_{3 z}}{2} \quad \text { if } \quad \mathrm{w}(\mathrm{i})=1
$$

$$
v_{2 x}^{\prime}=\frac{v_{0 x}+3 v_{3 x}}{4}, v_{2 y}^{\prime}=\frac{v_{0 y}+3 v_{3 y}}{4}, \quad v_{2 z}^{\prime}=\frac{v_{0 z}+3 v_{3 z}}{4} \quad \text { if } \quad \mathrm{w}(\mathrm{i})=0
$$

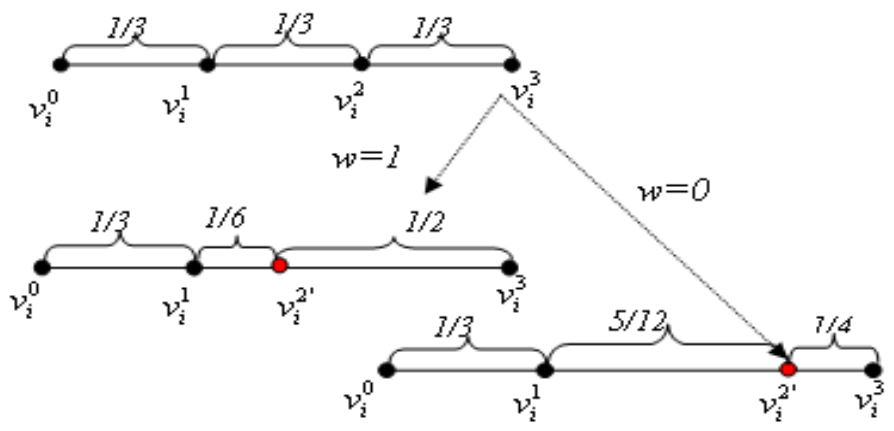

Figure 3. Embed a Watermark Bit into Line Entity $v_{i}^{0} v_{i}^{3}$

4.2.2 Embedding Watermark bits into Arc Entity: The method of embedding watermark bit into Arc entity is similar to Line entity. The only difference is that the angle of Arc $v_{i}^{1} v_{i}^{2}$ takes the place of the length of Line $v_{i}^{1} v_{i}^{2}$. 

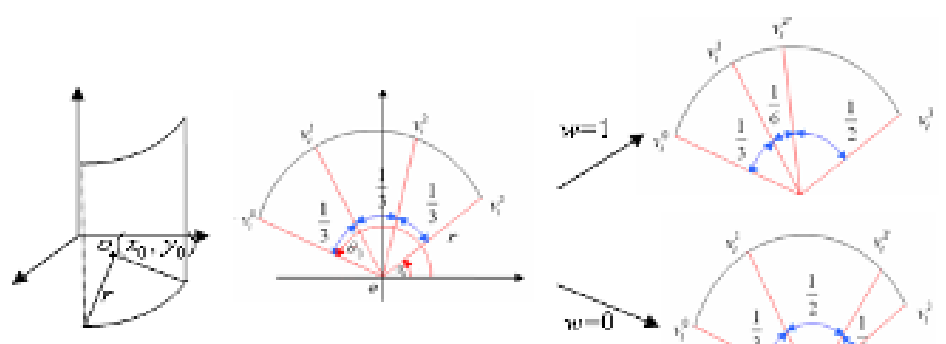

Figure 4. Embed Watermark into Arc Entity $v_{i}^{0} v_{i}^{3}$

Step 1: Arc entity $v_{i}^{0} v_{i}^{3}$ is projected into XY plane and the parametric equation of a circle is employed.

$$
v_{x}=x_{0}+r \cos \theta \quad v_{y}=y_{0}+r \sin \theta
$$

Here, $o\left(x_{0}, y_{0}\right)$ is the coordinate of the center of the circle, and $r$ is the radius.

Step 2: Arc entity $v_{i}^{0} v_{i}^{3}$ is divided evenly into three Arc entities. Calculate the coordinates of additional redundant points $v_{i}^{1}\left(v_{1 x}, v_{1 y}\right)$ and $v_{i}^{2}\left(v_{2 x}, v_{2 y}\right)$.

$$
\begin{aligned}
& \left\{\begin{array}{l}
v_{1 x}=x_{0}+r \cos \frac{\theta_{3}-2 \theta_{0}}{3} \\
v_{1 y}=y_{0}+r \sin \frac{\theta_{3}-2 \theta_{0}}{3}
\end{array}\right. \\
& \left\{\begin{array}{l}
v_{2 x}=x_{0}+r \cos \frac{2 \theta_{3}+\theta_{0}}{3} \\
v_{2 y}=y_{0}+r \sin \frac{2 \theta_{3}+\theta_{0}}{3}
\end{array}\right.
\end{aligned}
$$

Step3: adjust the location of $v_{i}^{2}\left(v_{2 x}, v_{2 y}\right)$ according to watermarking bit w(i) using the following equations and get as shown in Fig 4 .

$$
\begin{aligned}
& \left\{\begin{array}{lll}
v_{2 x}^{\prime}=x_{0}+r \cos \frac{\theta_{3}+\theta_{0}}{3} & \text { if } & w(i)=1 \\
v_{2 y}^{\prime}=y_{0}+r \sin \frac{\theta_{3}+\theta_{0}}{3} & \text { if } & w(i)=0
\end{array}\right. \\
& \left\{\begin{array}{l}
v_{2 x}^{\prime}=x_{0}+r \cos \frac{\theta_{3}+5 \theta_{0}}{6} \\
v_{2 y}^{\prime}=y_{0}+r \sin \frac{\theta_{3}+5 \theta_{0}}{6}
\end{array}\right.
\end{aligned}
$$

\subsection{Watermark Extracted}

The proposed scheme for watermark extraction is a blind watermarking algorithm. To extract the watermark, the original watermarked model and complicated pre-processing (registration or re-sampling), which affect the quality of the extracted watermark and make the non-blind watermarking impractical, need not be provided. Thus, our scheme is more practical. 
4.3.1Extracting Watermark Bits from Line/Entity: Watermarking information is extracted according to relative length of a line entity $v_{i}^{m-1} v_{i}^{m}$ to $v_{i}^{0} v_{i}^{k}$. If the length $v_{i}^{m-1} v_{i}^{m}$ is less than one-third of the length $v_{i}^{0} v_{i}^{k}$, we will assign 1 to w(i). Otherwise, we will assign 0 to $\mathrm{w}(\mathrm{i})$.

$$
\mathrm{w}(\mathrm{i})=1 \quad \text { if } \quad\left|v_{i}^{m-1} v_{i}^{m}\right|<\left|v_{i}^{0} v_{i}^{k}\right| / 3
$$

$$
\mathrm{w}(\mathrm{i})=0 \quad \text { if } \quad\left|v_{i}^{m-1} v_{i}^{m}\right|>\left|v_{i}^{0} v_{i}^{k}\right| / 3
$$

Here, $2 \leq m \leq k-1, \mathrm{k}=3$

4.3.2 Extracting Watermark Bits from Arc Entity: The scheme of extracting watermark bits from Arc entity is similar to the method of Line entity. The main difference between them is that the angle of circular arc takes the place of length of line.

$$
\begin{array}{lll}
\mathrm{w}(\mathrm{i})=1 & \text { if } & \angle v_{i}^{m-1} v_{i}^{m}<\angle v_{i}^{0} v_{i}^{k} / 3 \\
\mathrm{w}(\mathrm{i})=0 & \text { if } & \angle v_{i}^{m-1} v_{i}^{m}>\angle v_{i}^{0} v_{i}^{k} / 3
\end{array}
$$

Here, $\quad 2 \leq m \leq k-1, \mathrm{k}=3 . \quad \angle v_{i}^{m-1} v_{i}^{m}$ is the angle of arc $v_{i}^{m-1} v_{i}^{m}$

\subsection{Correlation Coefficient}

Compute its correlation coefficient $N C_{q}$ (q=line or q=arc) with the original watermark W,

$$
N C_{q}=\frac{\sum_{j=1}^{m} w_{i} \times w_{i}^{\prime}}{\sqrt{\sum_{j=1}^{m}\left(w_{i}\right)^{2}} \times \sqrt{\sum_{j=1}^{m}\left(w_{i}^{\prime}\right)^{2}}}
$$

In order to set an appropriate threshold value, we have done some experiments on the threshold. By generating 2000 random watermarks, each of them is a 100 random number sequence, and the 1000th is the watermark information that is used in our algorithm. We find the maximum correlation value is about 0.4 . For declining the probability of error, we can set the threshold value to be 0.5, as shown in Figure 5. In our algorithm, correlation coefficient $N C=\operatorname{Max}\left(N C_{\text {line }}, N C_{\text {arc }}\right)$

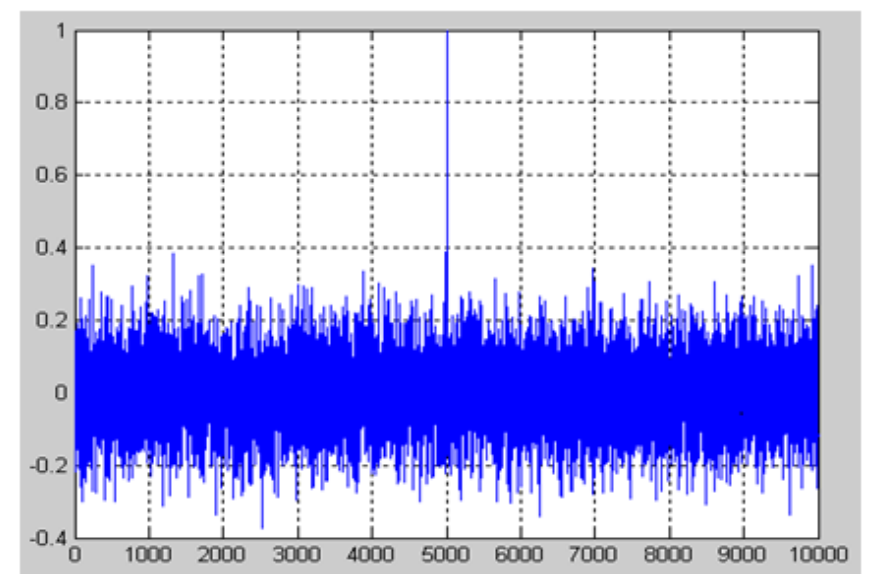

Figure 5. Experiments of Correlation Coefficient 


\section{Experiments and Results}

Experiments are carried out with Windows XP SP2, Solidworks 2006, and Matlab 7.0. Three 3D CAD product models (in IGES format) are used.

\subsection{Analysis of Imperceptibility}

In order to evaluate the distortion caused due to watermark embedding. The root mean square error (RMSE), as formulated in Eq. (15), is used in this watermarking scheme.

$$
R M S E=\frac{1}{N}\left\|V-V^{w}\right\|
$$

Here, $V$ and $V^{w}$ are the sets of vertices in the original 3D CAD product model and its watermarked model respectively, and $N$ is the number of vertices. The experimental results are listed in Table 1. Experiments show that our scheme will not bring any distortion to product model, which can keep strictly geometrical shape around embedding watermark and make the watermarked model have function transparency.

\subsection{Watermark Capacity}

Methods of modifying coordinate of vertex to embed watermark will cause distortion on the model. So, the relationship between watermark capacity and robustness needs to be considered. However, in our watermarking algorithm, the watermark information is embedded by substituting isolated geometrical entity. Furthermore, this scheme will not produce any distortion. Therefore the watermark capacity $\$ M \$$ is determined by the number of effective isolated geometric entities including Line and Arc $M=$ Mline + Marc

\section{Table 1. The RMSE Values between the Original 3D CAD Product Model and the Watermarked Models}

\begin{tabular}{|l|l|l|l|l|}
\hline 3D CAD product model & $\begin{array}{l}\text { Number of } \\
\text { entities }\end{array}$ & $\begin{array}{l}\text { Number of isolated } \\
\text { Line entities }\end{array}$ & $\begin{array}{l}\text { Number of isolated } \\
\text { Arc entities }\end{array}$ & RMSE \\
\hline 436 & 80 & 46 & 0.0 \\
\hline \begin{tabular}{l} 
Model1 \\
\hline Model2
\end{tabular} & 889 & 64 & 102 & 0.0 \\
\hline \\
\hline
\end{tabular}

\subsection{Robustness Evaluation}

For 3D CAD product model, translation, rotation, and scaling are typical operations in applications. Robustness against these operations is very important for a reversible watermark scheme. Experiments are done to analyze the robustness of the proposed 
scheme. As seen from the correlation coefficient $N C$ values listed in Table 2, the proposed methods are robust against translation, rotation, scaling and its combination.

5.3.1 Analysis of the Robustness against Similarity Transformations: Similarity transformation includes translation, rotation and scaling, and combinations of the above three operations. Similarity transformation is considered to be a common operation rather than an attack, against which even a fragile watermark should be able to stand.

5.3.2 Entity Deletion: In entity deletion experiments, we have done some experiments under different ratios of deleted entities to watermarked product models. Experimental result indicated that our algorithm is robust against entity deletion, as shown in Table 2. Figure 6 shows the experimental analysis results under different ratios of deleted entities. If there are only a few isolated geometric entities, the failure of extracting watermark would occur. Models 2 and 3 could not be used to extract watermark from Line entities when deleting 50\% entities, as shown in Fig. 6(a). However, Models 2 and 3 could be used to extract watermark from arc entities, as shown in Fig. 6(b). In order to avoid this

kind of error detection, we use the maximum method of $N C=\operatorname{Max}\left(N C_{\text {line }}, N C_{\text {arc }}\right)$ to judge existing of watermark, which can effectively reduce the false negative rate, as shown in Figure 6(c).

Table 2. Experiments of Entity Deletion

\begin{tabular}{|c|c|c|c|}
\hline Watermarked model & Ratio of entities deletion & NC & $\begin{array}{l}\text { Attacked } \\
\text { model }\end{array}$ \\
\hline \multirow[t]{3}{*}{ Model1 } & $20 \%$ & $\begin{array}{l}\mathrm{NC}=0.9530 \\
\left(\mathrm{NC}_{\text {line }}=0.7170\right) \\
\left(\mathrm{NC}_{\text {arc }}=0.9530\right)\end{array}$ & \\
\hline & $30 \%$ & $\begin{array}{l}\mathrm{NC}=0.9042 \\
(\mathrm{NC} \text { line }=0.6656) \\
\left(N C_{\text {arc }}=0.9042\right)\end{array}$ & \\
\hline & $50 \%$ & $\begin{array}{l}\mathrm{NC}=.0 .8572 \\
(\mathrm{NC} \text { line }=0.2793) \\
\left(\mathrm{NC}_{\text {arc }}=0.8572\right)\end{array}$ & \\
\hline \multirow{2}{*}{ Model2 } & $20 \%$ & $\begin{array}{l}\mathrm{NC}=0.8348 \\
(\mathrm{NCline}=0.8348) \\
(\mathrm{NCarc}=0.7933)\end{array}$ & \\
\hline & $50 \%$ & $\begin{array}{l}\mathrm{NC}=0.5462 \\
(\mathrm{NCline}=0.5462) \\
(\mathrm{NCarc}=0.5418)\end{array}$ & \\
\hline
\end{tabular}




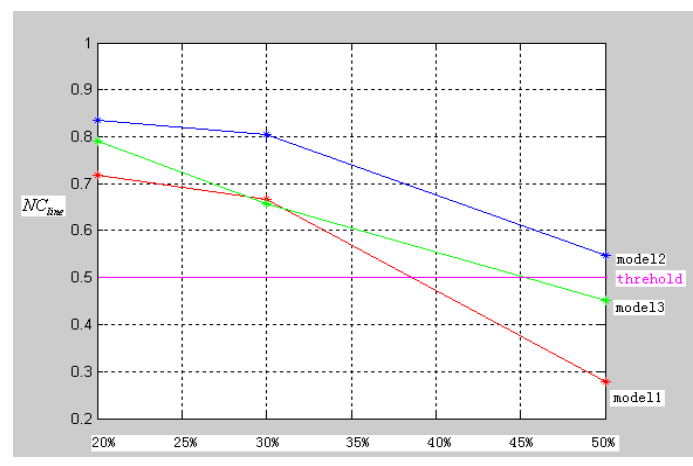

(a) $N C_{\text {line }}$

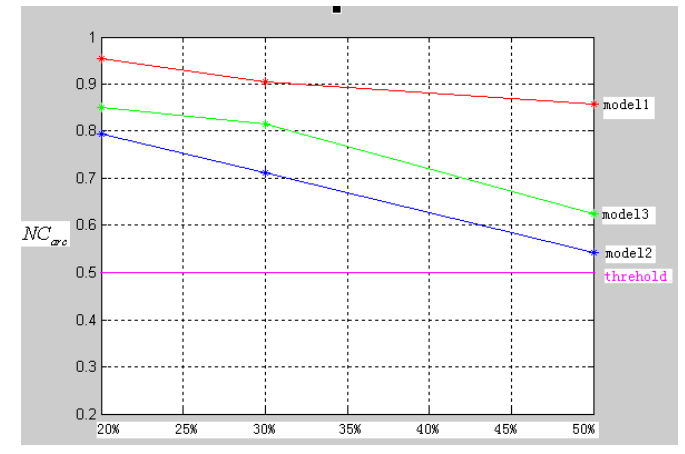

(b)

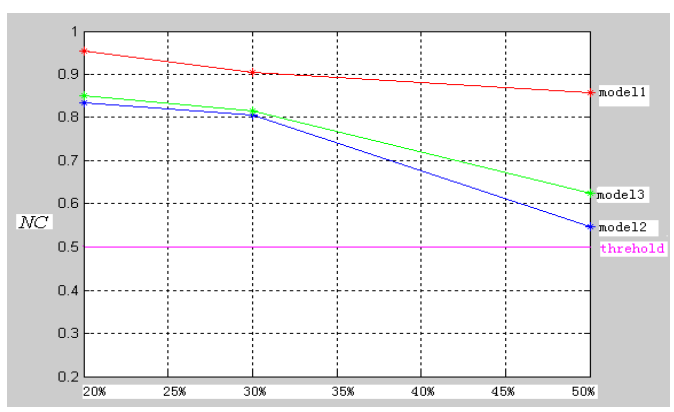

(c) $N C=\operatorname{Max}\left(N C_{\text {line }}, N C_{\text {arc }}\right)$

Figure 6. Experimental Analysis Results under Different Ratios of Deleted Entities

5.3.3 Time Complexity: Time complexity is an important evaluation index of an algorithm. The estimated time complexity of our algorithm for geometrical entity substitution is $O(n)$ (n is the number of isolated geometric entities). Experiments were done to analyse the relation between the time cost of embedding or extraction and the numbers of isolated geometric entity. As shown in the Figure 7, the time of embedding and extraction will be increased with the increase of numbers of isolated geometric entities. Furthermore, time of embedding will be increased more quickly than extraction. For user, the efficiency of watermarking algorithm depends mainly on extraction time. So, our scheme has good practicability.

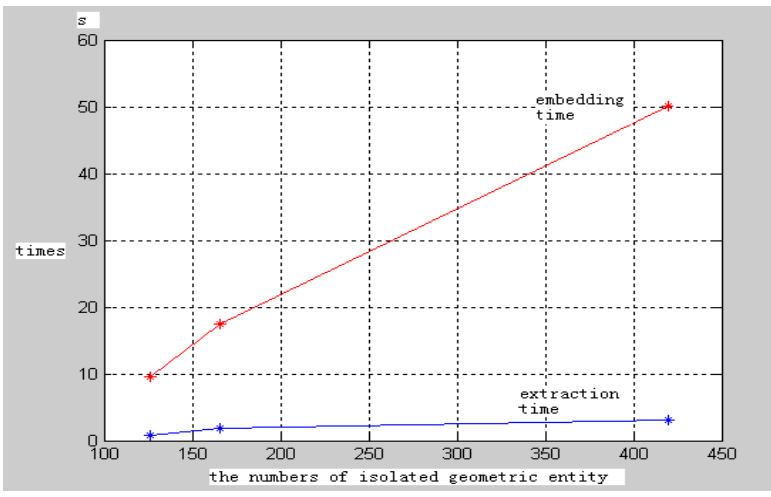

Figure 7. The Relationship between the Time Cost of Embedding or Extraction and the Numbers of Isolated Geometric Entity 


\section{Conclusion}

In the proposed blind watermarking scheme of 3D CAD product model, some isolated geometric entities are substituted with three geometric entities, and some additional vertexes are resulted. Watermark information is embedded by adjusting the location of additional vertexes along the track of the original geometrical entity according to each watermark bit. This scheme will not produce any distortion to the product model and can keep strictly geometrical shape around embedding watermark, which make the watermarked model have function transparency. Moreover, our watermark algorithm is a blind method. During extraction of watermark information, the original model and pre-process need not be provided. This means that it is adaptable for practical application of CAD product based on feature modeling. Finally, experiments show that the proposed algorithm is robust against some attacks, such as similarity transformation, entity deletion. However, it is worth noting that it is fragile against file format conversion.

In our future work, we will explore some other technologies such as feature trees and integrate them with our currently proposed approach in order to resolve the fragility of watermarking algorithm against file format conversion.

\section{Acknowledgement}

This work is supported by the National Natural Science Foundation of China (No. 61202197) and (No.61100084). Zhejiang Provincial Natural Science Foundation of China (No.LQ13F020003)

\section{References}

[1] S. Kanai, H. Date, and T. Kishinami, "Digital watermarking for 3D polygons using multiresolution wavelet decomposition", In: Processings of the Sixth IFIP WG 5.2 International Workshop on Geometric Modeling: Fundamentals and Applications (GEO-6), Tokyo, Japan, (1998), pp. 296-307.

[2] H. K. Chen and Y. H. Chen, "Progressive watermarking on 3D meshes", In: Proceedings of IEEE International Symposium on Broadband Multimedia Systems and Broadcasting. Shanghai, China, (2010), pp. 1-7.

[3] L. Li, D. Zhang, Z. G. Pan, J. Y. Shi, K. Zhou, and K. Ye, "Watermarking 3D mesh byspherical parameterization", Computers \& Graphics, vol. 28, (2004), pp. 981-989.

[4] A. G. Bors, "Watermarking mesh-based representations of 3D objects using local moments", IEEE T IMAGE PROCESS, vol. 15, no. 3, (2006), pp. 687-701.

[5] Y. P. Wang and S. M. Hu, "A new watermarking method for 3D models based on integral invariants", IEEE T VIS COMPUT GR, vol. 15, no. 2, (2009), pp. 285-294.

[6] W. Kai, L. Guillaume, D. Florence, and B. Atilla, "Robust and blind mesh watermarking based on volume moments", Computers \& Graphics, vol. 35, (2011), pp. 1-19.

[7] R. Ohbuchi, H. Masuda, and M. Aono, "Watermarking three-dimensional polygonal models", In: Proceeding of ACM Int. Multimedia Conf. and Exhib., (1997), pp. 261-272.

[8] Q. S. Ai, Q. Liu, Z. D. Zhou, L. Yang, and S. Q. Xie, "A new digital watermarking scheme for 3D triangular mesh models", Signal Process, vol. 89, no. 11, (2009), pp. 2159-2170.

[9] R. H. Juan, A. Martion, and P. G. Fernando, "DCT-domain watermarking techniques for still images: detector performance analysis and a new structure", IEEE T IMAGE PROCESS, vol. 9, no. 1, (2000), pp. 55-68.

[10] J. W. Cho, R. Prost, and H. Y. Jung, "An oblivious watermarking for 3-D polygonal meshes using distribution of vertex norms”, IEEE Trans. Signal Process, vol. 55, no. 1, (2007), pp. 142-155.

[11] X. Q. Feng, W. Y. Zhang, and Y. N. Liu, "Double watermarks of 3D mesh model based on feature segmentation and redundancy information", MULTIMED TOOLS APPL: DOI 10.1007/s11042-012-1039-7, (2012).

[12] K. R. Kwon, H. J. Chang, G. S. Jung, K. S. Moon, and S. H. Lee,"3D CAD drawing watermarking based on three components", In: Proceeding of IEEE International Conference on Image Processing, (2006), pp. 1385-1388.

[13] Z. C. Cai, W. Sun, C. Z. Xiong, and D. X. Qi, "Watermarking of two-dimensional engineering graphic based on the orthogonal complete U-system", In: 9th international conference on computer aided design and computer graphics. Hong Kong, China, (2005), pp. 205-209.

[14] X. F. Huang, F. Peng, and T. Deng, "A Capacity Variable Watermarking algorithm for 2D engineering graphic based on complex number system", In: Proceeding of Intelligent Information Hiding and Multimedia Signal Processing, IIHMSP '08, (2008), pp. 339-342. 
[15] F. Peng, Y.Z. Lei, M. Long, and X. M. Sun, “A reversible watermarking scheme for two-dimensional CAD engineering graphics based on improved difference expansion", COMPUT AIDED DESIGN, vol. 43, no. 8, (2011), pp. 1018-1024.

[16] S. H. Lee and K. R. Kwon, "CAD drawing watermarking scheme', DIGIT SIGNAL PROCESS, vol. 20, no. 5, (2010), pp. 1379-1399.

\section{Authors}

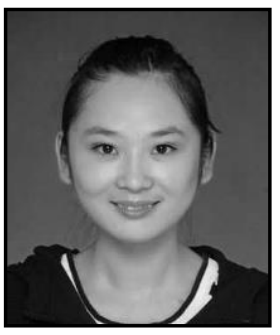

Xiaoqing Feng (1983-), College of Information, Zhejiang University of Finance \& Economics, Hangzhou, 310018, China.

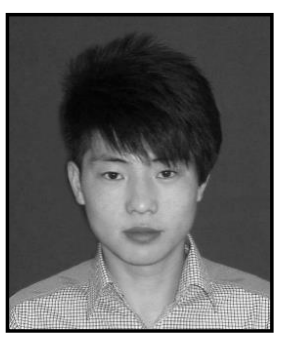

Yanan Liu (1982-) College of Information, Zhejiang University of Finance \& Economics, Hangzhou, 310018 , China. 
International Journal of Security and Its Applications

Vol.9, No.9 (2015) 